\title{
Medical Image Quantization using Biogeography based Optimization
}

\author{
Rajwinder Kaur \\ IET ,Bhaddal, \\ Punjab, India
}

\author{
Rakesh Khanna \\ Assistant Professor \\ IET, Bhaddal, \\ Punjab, India
}

\begin{abstract}
Biogeography based optimization (BBO) is a type of evolutionary algorithm. It is a population based optimization algorithm and provides clarification about the changing distribution of all species in different environment with time. Color quantization is the process of reducing the number of colors in the image and preserving the most important color information and compromise with other. A color image quantization is necessary if the display on which a specific image is presented works with less colors then the original image. Color quantization plays an important role in many medical imaging. This paper provides methods for quantization of various medical images like MRI, X-Ray using Biogeography Based optimization technique.
\end{abstract}

\section{General Terms}

Habitats, HSI, SIV, Immigration rate, Emigration rate, HSV, CIEL*a*b, CIEL*u*v

\section{Keywords}

BBO, Quantization, anatomical medical images, evolutionary algorithm.

\section{INTRODUCTION}

Medical imaging is an effective process of creating the image of internal human body parts. Medical imaging techniques produce very large data because of which communication and storage of data is very difficult. In order to remove this drawback compression of images is done. Another advantage of compressing these images is their low cost and feasibility to produce large images over low bandwidths. There are various techniques that have been used till now for compression of medical images. Color quantization is one of the techniques that is used worldwide for compression of image.

Color quantization technique is used to reduce the number of distinct colors in an image, but the new image formed should be visually similar to the original image. It helps in reducing the memory requirement of the images. For implementation of color quantization technique the first requirement is to find out the actual number of colors present in the image. We can choose any color model like RGB, LAB and CMY etc. for it. Then the color distance is measured using different types of distance formula techniques. Then the mapping of close colors is done and the far off colors are dropped. Based on our requirement we can decide the final number of colors in the image and can stop further reduction of colors. The process of quantization should not cause any loss to the visual information of the images but it should reduce its memory requirements. The algorithm of color mapping can be divided into two classes splitting algorithm and clustering based algorithm.
Medical image segmentation [1] is the first step in many medical image analysis and quantization methods. In previous years, medical image segmentation was an important area of interest for researchers as manual segmentation of medical images was almost impossible. After segmentation of medical images, quantization of images is done. This paper explains how color quantization of medical images is done using BBO, it is a population algorithm where histograms of equally sized ranges are constructed and colors are assigned to the ranges containing maximum points.

\subsection{Biogeography based optimization}

Biogeography is the study of geographical distribution of biological organisms. As the name implies, BBO is based on the mathematical study of biogeography. It is the study of the distribution of animals and plants over time and space. The aim of this study is to provide clarification about the changing distribution of all the species [6] in different environments over time. (Haiping Suhong and Man, 2009).

Mathematical models of biogeography describes how species migrate from one island to another, and how new species arise and become extinct. An island is a habitat that is geographically isolated from other habitats. Geographical areas that are well suited for the residence of biological species are said to have high suitability index (HSI) [2] and the variables that characterize habitability are called suitability index variables (SIV) [4].

Habitats with high HSI have large number of species, while those with low HSI have smaller number of species. Habitats with high HSI have many species that tend to emigrate to near habitats and they have a low species immigration rate because they are already saturated with species. Therefore, high HSI habitats are more stable in their species distribution than low HSI habitats. Habitats with a low HSI have a high species immigration rate because of large population. Thus immigration of new species to low HSI habitats may increase the HSI of the habitat, because the suitability index of a habitat is proportional to its biological diversity. However, if habitats HSI remains low, then the species that reside there will tend to get extinct, which will further cause additional immigration. Due to this low HSI, habitats are more unstable in their species distribution than high HSI habitats. 




Fig1. Migration of species.

\section{BBO consist of following basic steps:}

\subsubsection{Migration}

Consider a population of candidate which is represented by design variable. Each design variable for particular population member is considered as suitability index variable (SIV) for that population member. Each population member is considered as individual habitat or island. The objective function value indicates the HSI for particular population member. Immigration and emigration rates are decided by the curves in figure 1 . The nature of the curve is assumed to be same for immigration and emigration rate but with opposite slopes as both are linear in nature. Value of $\mathrm{S}$ represented by the solution depends upon its HSI. The immigration rates and emigration rates both are used for sharing the information between the two habitats as required. If a solution is selected to be modified, then its immigration rate $\lambda$ is used to modify SIV in the solution. If a given SIV [8] in a given solution $S_{i}$ is selected to be modified, then emigration rate $\mu$ of the other solution is used to decide which of the solution should migrate to the randomly selected SIV of the solution $\mathrm{S}_{\mathrm{i} . .}$. This phenomenon is known as migration in Biogeography Based optimization.



Number of species

Fig2. Species of a single model

The BBO migration property is similar to the breeder Genetic Algorithm [5]. The evolutionary strategies and BBO differs by one important property. In evolutionary strategies, global recombination is used to create new solutions, but in BBO migration is used to change existing solutions .Global recombination is a reproductive process, whereas migration in $\mathrm{BBO}$ is an adaptive process so it is used to modify existing islands.

\subsubsection{Mutation}

In nature a habitat's HSI can change suddenly due to random events. This phenomenon is known as SIV mutation and probabilities of species count are used for finding mutation rates. This probability mutates low HSI as well as high HSI solutions. Equation used for finding mutation rate is as follows.

$$
\mathrm{M}(\mathrm{S})=\mathrm{M}_{\max }\left(1-\frac{P_{S}}{P_{\max }}\right)
$$

Where $\mathrm{M}_{\max }$ is a user defined parameter called mutation coefficient. The mutation mechanism depends upon given problem as in genetic algorithm.

\section{PROPOSED ALGORITHM}

Medical image segmentation is the $1^{\text {st }}$ step in many medical image segmentation and quantization methods. Segmentation of medical images involves three stages.

\subsection{Noise removal}

As image contains noise which can change the intensity of the pixel. Therefore, it is desirable to perform some kind of noise removal technique on an image. The median filter is a non linear digital filtering technique used to remove noise. It is basically a pre-processing process to improve the result of the later processing. After noise removal edge detection of image is done.

\subsection{Edge detection of medical image}

Edge detection [7] of image is done using any of the one approach given below.

\subsubsection{The Marr-Hildreth edge detector}

It is a very popular gradient based edge operator. It uses the laplacian operator to take the second derivative of an image. If there is a step difference in the intensity of the image, it will be represented by second derivative by zero crossing.

\subsubsection{The canny edge detector}

This detector is widely used for standard edge detection algorithm in industry. The main steps are: Smooth the image with a two dimensional Gaussian, then take the gradient of the image. This shows change in intensity, which indicates the presence of edges. This gives two results, the gradient in the $x$ direction and the gradient in the y direction. Edges will occur at points where the gradient is at its maximum.

\subsubsection{Morphological edge detection}

Morphological edge detection technique selects appropriate structuring element of the processed image. This detection technique makes use of the basic theory of morphology including erosion, dilation, opening and closing operation and the synthesis operation to get a clear image.

\subsubsection{Color edge detection using Euclidean distance and vector angle}

It uses two operators: Euclidean distance and vector angles. These operators are used to detect edges in regions with high colour variations and are therefore selected for solving this problem. Euclidean distance is a good operator for finding edges based on hue and saturation. The detector applies both operators to the RGB color space of an image, and then combines the result based on the amount of color in a region.

The algorithm for finding edges in an image is as follows:

- For each pixel in the image, take the $3 \times 3$ window of pixel. 
- Calculate the Euclidean distance and vector angle between the centre point and the points surrounding it.

- Assign the largest value obtained to the centre pixel.

- When each pixel has a value assigned to it, run the results through a threshold to eliminate false edges.

\subsection{Color quantization of color space model using BBO approach}

The purpose of color space model [10] is to facilitate the specification of colors. Each color in the color space is a single point represented in the coordinate system. For the retrieval of medical images, color allows images to reveal many morphological and pathological characteristics. Several color spaces such as $R G B, H S V, C I E L^{*} a * b$ and $C I E L * u * v$, have been developed for different purposes. The most frequently used technique is to convert color representation from the RGB color space to the $H S V, C I E L^{*} u^{*} v$ or $C I E L * a * b$ color spaces with perceptual uniformity. Color system is very useful in interactive color selection and manipulation. The $C I E L^{*} u^{*} v$ and $C I E L * a * b$ both are perceptually uniform systems, which provides easy way of similar metrics for comparing colors.

$C I E L * a * b$ space uses lightness (L), red-greenness (a), and yellow blueness (b) as its tristimulus values. CIEL*a*b [3] is the most complete color model used conventionally to describe all the colors visible to human eye. It was developed for this specific purpose by the international commission on illumination. It made adjustment to the CIEXYZ color space to make it perceptually linear so that the color changes at a constant rate. For each of the absolute spaces there are techniques for converting to and from CIEXYZ absolute color space.

$L^{*}=116 f\left(Y / Y_{n}\right)-16$

$a^{*}=500\left[f\left(X / X_{n}\right)-f\left(Y / Y_{n}\right)\right]$

$b^{*}=200\left[f\left(Y / Y_{n}\right)-f\left(Z / Z_{n}\right)\right]$

\section{Where}

$f(t)=t^{1 / 3}$ for $t>0.008856$

$f(t)=7.787 t+16 / 116$ otherwise.

Here $X_{n}, Y_{n}, Z_{n}$ are the CIEXYZ tristimulus values of the reference white point.

In this study, we used LAB color model for calculation of CMC [12] distance as it gives better results than any other model. CMC stands for color measurement committee of the society of dyers and colorists. It has two parameters: lightness (l) and chroma (c), allowing the user to weight the difference based on the ratio of $1: c$ that is appropriate for this application. The distance of the color $\left(\mathrm{L}_{2}{ }^{*}, \mathrm{C}_{2}{ }^{*}, \mathrm{~h}_{2}\right)$ to the reference $\left(\mathrm{L}_{1}{ }^{*}, \mathrm{C}_{1}{ }^{*}, \mathrm{~h}_{1}\right)$ can be calculate using formula:

$\Delta E_{C M C}^{*}=\sqrt{\left(\frac{L_{2}^{*}-L_{1}^{*}}{l S_{L}}\right)^{2}+\left(\frac{C_{2}^{*}-C_{1}^{*}}{c S_{c}}\right)^{2}+\left(\frac{\Delta H_{a b}^{*}}{S_{H}}\right)^{2}}$
$S_{L}=\left\{\begin{array}{lc}0.511 & L_{1}^{*}<16 \\ \frac{0.040975 L_{1}^{*}}{1+0.01765 L_{1}^{*}} & L_{1}^{*} \geq 16\end{array}\right.$

$S_{C}=\frac{0.0638 \mathrm{C}_{1}^{*}}{1+0.0131 \mathrm{C}_{1}^{*}}+0.638$

$S_{H}=S_{C}(F T+1-F)$

$F=\sqrt{\frac{C_{1}^{* 4}}{C_{1}^{* 4}+1900}}$

$T=\left\{\begin{array}{lr}0.56+\left|0.2 \cos \left(h_{1}+168^{0}\right)\right| & 164^{0} \leq h_{1} \leq 345^{0} \\ 0.36+\left|0.4 \cos \left(h_{1}+35^{\circ}\right)\right| & \text { otherwise }\end{array}\right.$

The proposed algorithm is as follows:

1. Convert a medical image into LAB image.

2. Calculate $\mathrm{CMC}$ distance between neighbouring pixels.

3. Initialise the BBO parameters, considering every color as a different habitat and every pixel as species.

4. Initialise a random set of habitats. Each habitat corresponds to a potential solution.

5. Select color represented by maximum number of pixels as $S_{\max }$ and the color represented by minimum number of pixels as $S_{\min }$.

6. For each habitat map the HSI to the number of species.

- Consider habitats with number of pixels lying in the range of $S_{\min }$ to $1 / 3$ of $S_{\max }$ as the habitats with low HSI.

- Habitats with number of pixels lying in the range of $1 / 3$ of $S_{\max }$ to $2 / 3$ of $S_{\max }$ as the habitats with moderate HSI.

- Habitats with number of pixels lying in the range of $2 / 3$ of $S_{\max }$ to $S_{\max }$ as the habitats with high HSI.

7. The immigration rate $\Lambda$ and emigration rate $\mu$ is calculated for each habitat. High HSI habitats have low emigration but high immigration, whereas low HSI habitats have low immigration but high emigration rate.

8. Probabistically use immigration and emigration to modify each non elite habitat, then recompute each HSI.

9. Now, for each habitat update the immigration and emigration rate of its species count using step 2.Increase the CMC distance for every iteration. Iterate till the required number of habitats are left.

\section{EXPERIMENTAL RESULTS \& DISCUSSION}

The proposed algorithm is used for quantization of medical images using Biogeography Based Optimization. Randomly selected images were color quantized. Following results are shown with original images and color quantized images. 


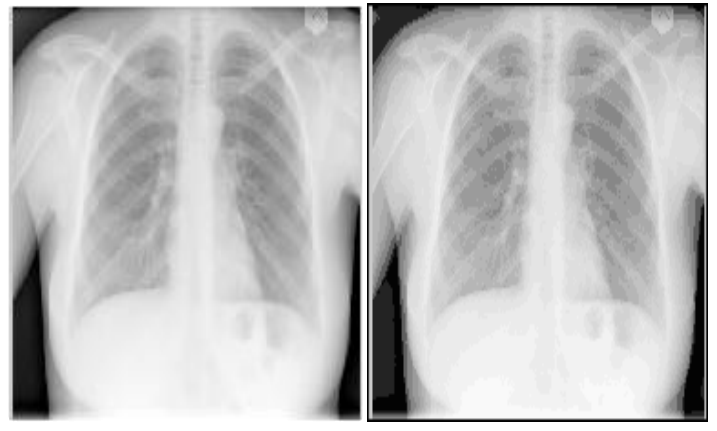

Fig 3 Original image and color quantized image

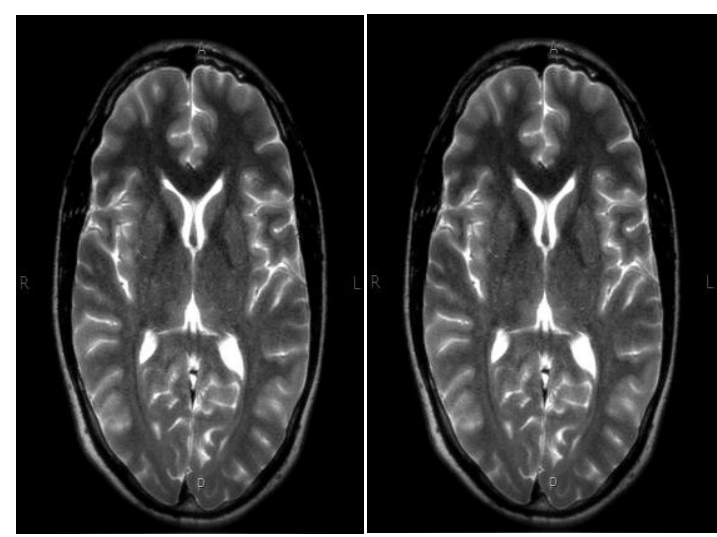

Fig 4 Original image and color quantized image

In earlier papers, work has been done on normal images but in this work we used medical images instead of normal images and checked whether medical data can be preserved using this technique of color quantization. The output of the above algorithm can be seen in the above figure.

\section{CONCLUSION}

In this paper BBO technique is applied for quantization of a anatomical medical image. Color quantization plays an important role while dealing with medical images. BBO technique is typically used for detection of abnormal growth of tissues. Manual quantization of these tissues cannot be compared with the fast day today methods. A randomly selected MRI database is used for testing. Major objective of this algorithm is to find the tumour area clearly and to check the kind of abnormalities if present.

\section{ACKNOWLEDGMENTS}

It gives me immense pleasure to express my deep sense of gratitude to Rakesh Khanna, Assistant Professor, IET Bhaddal for his able guidance with responsive encouragement, Constructive criticism without which it would have not been possible for me to complete my work.

\section{REFERENCES}

[1] Bandyopadhyay, S. K. 2011. "Colour quantization Technique for medical image segmentation", International Journal of research and reviews in computer science (IJRRCS), Vol. 2, No. 2, page 627631, April 2011.

[2] T. Wesche ,G.Goertler,and W.Hubert,"Modified habitat suitability index model for brown trout in southern eastern Wyoming", North Amer.J.Fisheries Manage.,vol 7,pp-232-237,1987.

[3] Hunter,Richard Sewall (July 1948)."Photoelectric color difference meter ".JOSA(proceeding of winter meeting of the optical society of America)

[4] Dan simon ,Senior member ,IEEE,IEE transactions on evolutionary computation ,Vol 12,No. 6,pp-702713,December 2008 .

[5] H. Muhlenbin and D.Schlierkamp -Voosen, "Predictive model for the breeder Genetic Algorithm: I.Continous parameter optimization",Evol. Comp.,vol. 1, pp-25-49, 1993

[6] Vitaliy F (2006), differential evolution in search of solutions. Springer, New York.

[7] Rajinder Kaur et al., "Color Image Quantization based on Bacteria Foraging Optimization", International Journal of Computer Applications (0975 - 8887) Volume 25- No.7, July 2011

[8] Surbhi Gupta et al., "Implementing Color Image Segmentation Using Biogeography Based Optimization", 2011 International Conference on Software and Computer Applications IPCSIT vol.9 (2011) () (2011) IACSIT Press, Singapore.

[9] Cheng, S.C., Yang, C.K., 2001. "A fast and novel technique for color quantization using reduction of color space dimensionality", Pattern Recognition Lett. 22, 845-856.

[10] Surbhi Gupta et al., "Color quantization in document images using biogeography based Optimization”, 2011 International Conference on Software and Computer Applications IPCSIT vol.9 (2011) (C) (2011) IACSIT Press, Singapore.

[11] Chia-Hung Wei, University of Warwick, UK et al. ,"A content based approach to medical image database retrieval",pp-258-291, wei,Li and Wilson.

[12] Seema Bansal et al., "Color Image segmentation using CIElab color space using Ant Colony optimization", International Journal of Computer Applications (0975 8887) Volume 29- No.9, September 2011. 